\title{
The investigation on defect recognition system using gaussian smoothing and template matching approach
}

\author{
M. H. Harun', M. F. Yaakub', A. F. Z. Abidin ${ }^{3}$, A. H. Azahar ${ }^{4}$, M. S. M. Aras ${ }^{5}$, M. B. N. Shah ${ }^{6}$, \\ M. F. M. Basar ${ }^{7}$ \\ 1,2,3,4,6,7Faculty of Electrical \& Electronic Engineering Technology, Universiti Teknikal Malaysia Melaka, Malaysia \\ ${ }^{5}$ Faculty of Electrical Engineering, Universiti Teknikal Malaysia Melaka, Malaysia
}

\begin{tabular}{l}
\hline Article Info \\
\hline Article history: \\
Received Sep 10, 2019 \\
Revised Nov 11, 2019 \\
Accepted Nov 25, 2019 \\
\hline
\end{tabular}

\section{Keywords:}

Gaussian smoothing

Gluing process

Region of interest

Shape-based matching

Template matching

\begin{abstract}
This paper investigates various approaches for automated inspection of gluing process using shape-based matching application. A new supervised defect detection approach to detect a class of defects in gluing application is proposed. Creating of region of interest in important region of object is discussed. Gaussian smoothing features is proposed in determining better image processing. Template matching in differentiates between reference and tested image are proposed. This scheme provides high computational savings and results in high defect detection recognition rate. The defects are broadly classified into three classes: 1) gap defect; 2) bumper defect; 3) bubble defect. This system does lessen execution time, yet additionally produce high precision in deformity location rate. It is discovered that the proposed framework can give precision at $95.77 \%$ recognition rate in recognizing imperfection for gluing application.
\end{abstract}

Copyright $\odot 2020$ Institute of Advanced Engineering and Science. All rights reserved.

\section{Corresponding Author:}

Mohamad Haniff Harun,

Faculty of Electrical \& Electronic Engineering Technology,

Universiti Teknikal Malaysia Melaka,

Hang Tuah Jaya, 76100 Durian Tunggal, Melaka, Malaysia.

Email: haniff@utem.edu.my

\section{INTRODUCTION}

Vision-based inspection of industrial products offers low-cost, high-speed, and high-quality detection of defects. One of the most challenging industrial inspection problems is dealing with the textured of the gluing process. Defects often happen during gluing process. To ensure high quality units are produced, defects detection and recognition system commonly are installed for segregation of the defects unit. There are many techniques that provide a solution in recognizing image or object in image processing such as region [1], edge-based features [2], feature extraction [3], shape context [4], Gaussian Curve [5] and etc. The work of image recognition based on HALCON application for shape-based matching is done in [6-10]. The researches discussed about the process involved in basic shape based matching algorithm together with Extended Region of Interest (ROI) function available in HALCON that fulfils shape based matching to find object based on a single model image with sub pixel accuracy. The basic concept of defect matching using shape-based matching algorithm based on Extended ROI introduced by [11].

In this paper, an improve filter method that uses a computer to automatically recognize the gluing defects is utilized. In this approach, the digital image of the gluing defects is binarized first. Then ROI is further process before. Correlation algorithm refines the image. Once the importance feature of the binarized image has been extracted, the features are trained for defect classification. To better understand this method, in the next section, each kind of defects is elaborated further. ROI is applied to reduce processing time. Then depending on the features classification of the defect, feature extraction and Gaussian smoothing are used. Not only that, the position of the camera must be accurate in order to get better images [12]. 
Minimal effort, rapid, and great discovery of deformities is the thing that vision-based assessment of modern items brings to the table. Issues managing the finished of the sticking procedure are the absolute most testing modern review. Deformities are regular happened in sticking procedure. Deformities identification and acknowledgment are most mainstream application used to affirm the uprightness of sticking procedure. Most scientists are for the most part unifying their reflection in welding line and furthermore in texture and many intriguing outcomes have been recovered [13-24]. For all intents and purposes, every one of these scientists can be delegated two sorts, one depends on radiographic investigation and the others dependent on man-made consciousness. The principal sort of strategies is to descry welding absconds physically, so the effectiveness is quite restricted [13-15]. The second sort of techniques are much outperforming in proficiency, anyway the unpredictability of execution is superfluous [16-24].

The other research is pinpointing on the welding imperfection $[20,24]$ that uses the component extraction strategy to rearrange picture to a straightforward calculation which depends on the perceptron model to descry and classify the lack as indicated by the information yielded from the extirpation technique. Picture of welding line is essential to the component destruction and deformity acknowledgment so as to perceive and characterize the welding deserts correctly.

\section{TYPES AND CHARACTERISTIC OF GLUING DEFECTS}

In this work, the study is performed to detect defects of gluing process at cardboard. Obviously, shape-based matching [25] requires reference image captured from the gluing process. The characteristic of each defect should be extract clearly as a model defect. The image obtained will undergo the pre-treatment of the images which include binarization processing, ROI, feature extraction, and Gaussian smoothing. Feature extraction processing is the important part that extracts the important features of each defect. This is because without proper feature extraction, the system cannot create high quality characteristic of the object. Without it, the recognition data will be affected.

As all digital images of gluing defects are binary images for defects and background. The gray level of defects divided into 255, while the background area is 0 . So when the gray level of all pixels of an image is 0 , then there are no defects in the gluing process based on the image. There are three kinds of defects altogether in gluing process which are gap defect, bumper defect and bubble defect. To begin with, the characteristic of these defects will be analyzed which will benefit for feature extraction as shown in Table 1.

Table 1. The Characteristic of Each Defect

\begin{tabular}{ccl}
\hline No. & Defect & \multicolumn{1}{c}{ Description } \\
\hline 1 & Gap Defect & $\begin{array}{l}\text { Gluing line surface that irregular, parallel empty space with gluing line, glue that } \\
\text { rugged until exposing space that not encrusted with glue. } \\
\text { Gluing line surface that irregular, swollen that similar to hump until deform } \\
\text { gluing line surface due to imbalance in object surface. } \\
\text { Gluing line surface that regular but is trapped air bubble in gluing line which } \\
\text { caused object surface not glue perfectly. }\end{array}$ \\
\hline
\end{tabular}

\section{OVERALL SYSTEM}

One of the applications of this research is to provide improved gluing car windscreen in automation industry. The system is developed to cope with the environment such as lighting condition, scaling and rotating of the object. Pyramid object developed from plain cardboard is created based on the shape object that has a contour that involves height, length and width. Plain cardboard is design exactly like pyramid to get the data from the Defect Shape Matching (DSM) whether the system able to recognize the object. Two cameras are used to locate $\mathrm{x}, \mathrm{y}$ and $\mathrm{z}$ location for each corner detected through the system. Equation of straight line will be used in determining additional point between two corners. Data gathered must be converted to robot coordinate for easier process by Katana and KUKA arm robot. This information then used to instruct robot for gluing purposes. Three types of defect are trained through Defect Inspection Monitoring (DIM) known as gap defect, bumper defect and bubble defect. Result obtained from DIM will decide whether the object is successful gluing or needs to be corrected through Correction of Defect (CoD). Figure 1 show the overall system of this research. 


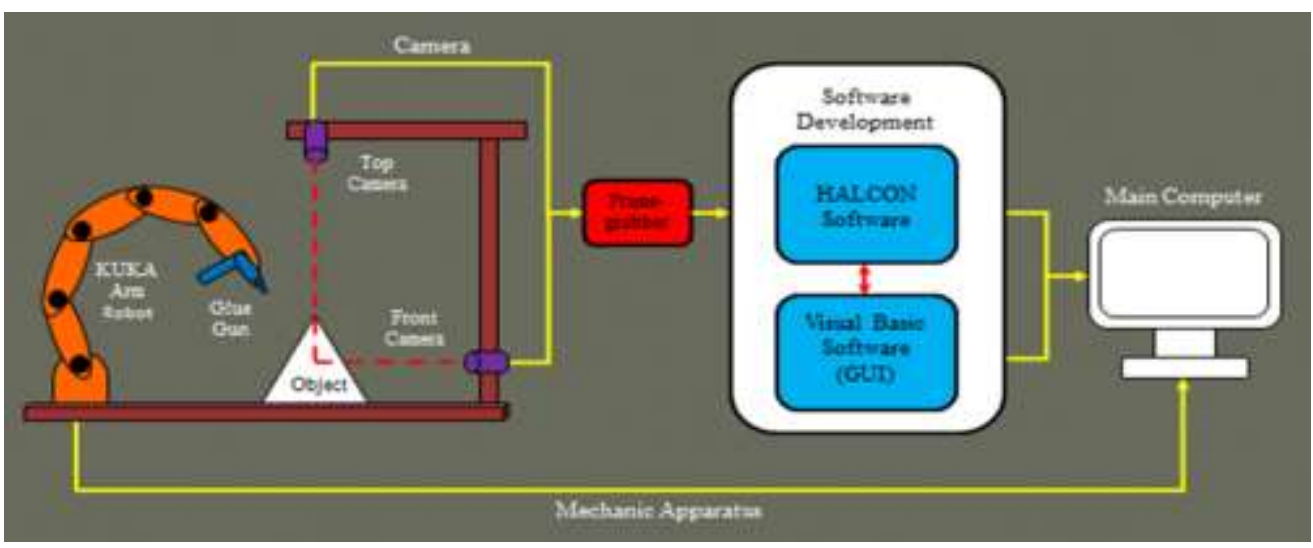

Figure 1. Proposed vision system

\section{SHAPE-BASED MATCHING}

Shape-based matching application divides into two phases namely as training phase and recognition phase [10] as shown in Figure 2. Training phase is a crucial part in identifying accurate defectiveness. Optimized training system will results in less accurate matching performance. Therefore, accurate matching results are depending to high quality reference image. The recognition phase is performed to find the similarity between reference images and tested images, where the similarity index in known as recognition rate. High recognition rate reflecting that, the proposed system is effective and optimized. Shape-based matching system is always favorable since the user can simply choose the desired region, fast processing time and provide higher recognition rate.

The proposed method consists of 4 main steps that valid in succession of classification glue defects: 1) Feature Extraction; 2) Gaussian smoothing; 3) Template creation; 4) Template matching. Introduction to this system further increase recognition rate and further accelerate inspection process. Furthermore, this proposed method develops with simplest features that only focused on images as an input and output.

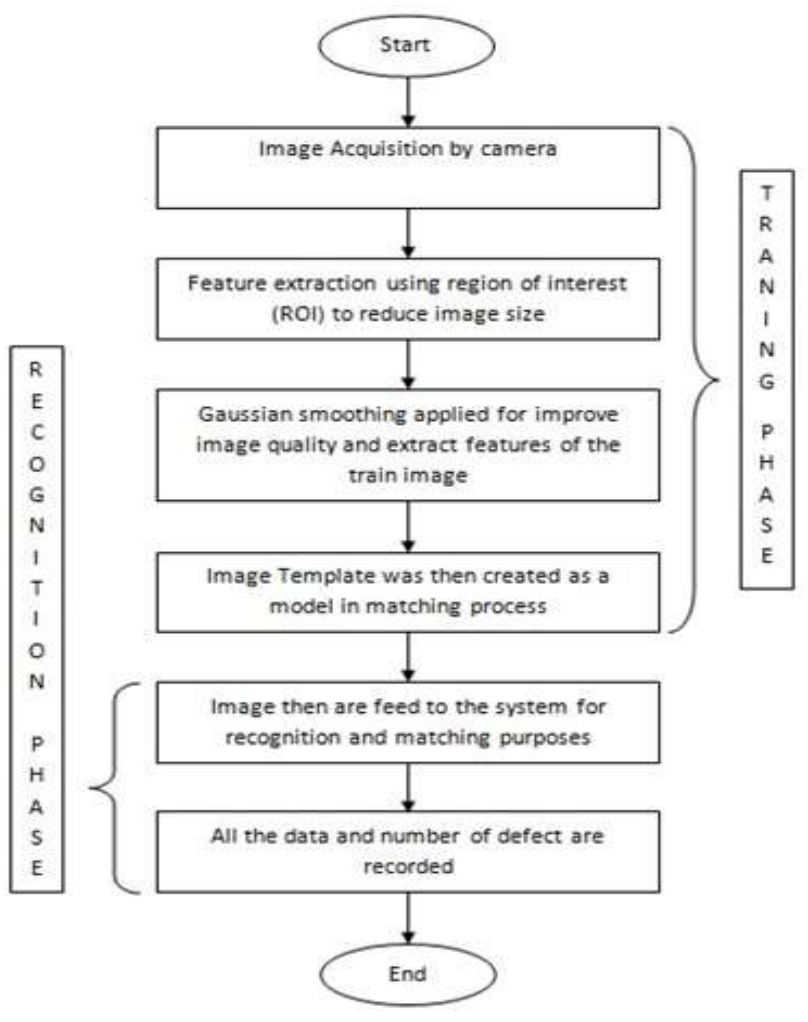

Figure 2. Proposed vision algorithm 


\section{FEATURE EXTRACTION}

To classify and recognize the defects mentioned Section 2, three features have been adopted. They are gray level, region of interest and converting skeleton to XLD contours.

\subsection{Gray Level}

To classify and recognize the defects mentioned above, three features has been adopted. There are gray levels, ROI and skeleton to XLD contours conversion. The grey level can be expressed as the following.

$$
\text { gray }=0.299 \alpha+0.587 \beta+0.144 \gamma
$$

Where $\alpha, \beta$ and $\gamma$ are red, green and blue respectively.

This feature affords in separating colors that may influence defect results. Gray level application selection helps in image of interest isolation with object background. The results facilitate system in developing defect model template.

\subsection{Region of Interest}

Region of interest (ROI) can be defined as a selected subset of samples within the dataset identified for a particular purpose known as boundary of an object. ROI provides the object to be match and its background as an input. This feature helps to minimize noise, improves matching speed and reduces matching parameter of the system since only the data within the selected ROI is used for the next process.

\subsection{Converting Skeleton to XLD Contours}

Changes over the info skeleton (e.g., edges), which is accepted that contain generally one-pixel wide areas, into XLD shapes. The district is first changed to contain just line fragments in 8-neighborhood. In the process 12 exceptional designs are considered: Focuses for which there is an intersection of at least three lines in 8-neighborhood is saved (in every one of the four turns)::

$$
\left(\begin{array}{lll}
0 & 0 & 1 \\
1 & 1 & 0 \\
0 & 1 & 0
\end{array}\right)\left(\begin{array}{lll}
0 & 1 & 0 \\
0 & 1 & 1 \\
0 & 1 & 0
\end{array}\right)\left(\begin{array}{lll}
0 & 1 & 0 \\
1 & 1 & 1 \\
0 & 1 & 0
\end{array}\right)
$$

In a subsequent advance, all intersection focuses are labeled, considering six distinctive trademark setups of each of the four potential pivots:

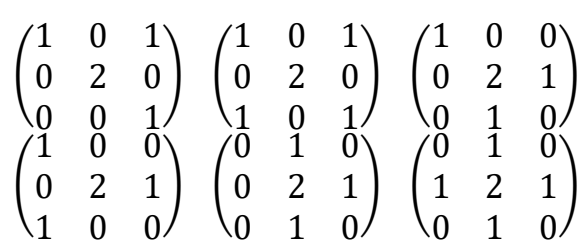

Where, $0=$ background, $1=$ foreground and $2=$ junction point.

Consequences of the two stages are for the most part forms having in any event length focuses are returned. The shapes created consistently end in intersection or end focuses. For shut shapes the main point lies in the 8-neighborhood of the last purpose of the form. In this manner, so as to decide the contiguousness of forms it is adequate to simply consider the end focuses.

The importance of this step provides better shape of the defects model. Not only that, time consuming in classified the defect may reduce.

\section{GAUSSIAN SMOOTHING}

The smoothing of gray images are using recursive and non-recursive Gaussian filter. There is available in four types of filter: 1) deriche1; 2) deriche2; 3) shen; 4) gauss. The channel width (i.e., the scope of the channel and in this manner consequence of the channel) of the picture can be of any size. For the situation that he Deriche or Shen is picked it diminishes by expanding the channel parameter alpha $(\alpha)$ and increments on account of the Gauss channel (and $\alpha$ compares to the standard deviation of the Gaussian capacity). An estimate of the proper size of the channel width is performed.

Non-recursive channel like the Gaussian channel are regularly executed utilizing channel veils. For this situation, the runtime of the administrator increments with expanding size of the channel cover. 
The runtime of the recursive channels stays steady: with the exception of the outskirt treatment turns into somewhat additional tedious. A practically identical aftereffect of the smoothing is accomplished by picking the accompanying qualities for the parameter:

$$
\begin{aligned}
& \alpha(\text { deriche } 2)=\frac{\alpha(\text { deriche } 1)}{2} \\
& \alpha(\text { shen })=\frac{\alpha(\text { deriche } 1)}{2} \\
& \alpha(\text { gauss })=\frac{1.77}{\alpha(\text { deriche } 1)}
\end{aligned}
$$

\section{TEMPLATE CREATION}

After image extracted using feature extraction and Gaussian smoothing, template creation is performed. Template creation is important in determining the originality of each defect characteristic to be submitted into the system for matching purposes. During the template creation step, the size of the required features must be considerably large to prevent the created outline does not overlap with the object and background can cause inaccuracy.

In order to achieve good template matching results, the pixel value difference between the object and the background must be sufficient for the system to work effectively. During the training phase, the resultants template must have an obvious shape. The training templates for this experiment are shown as example in Figure 3.

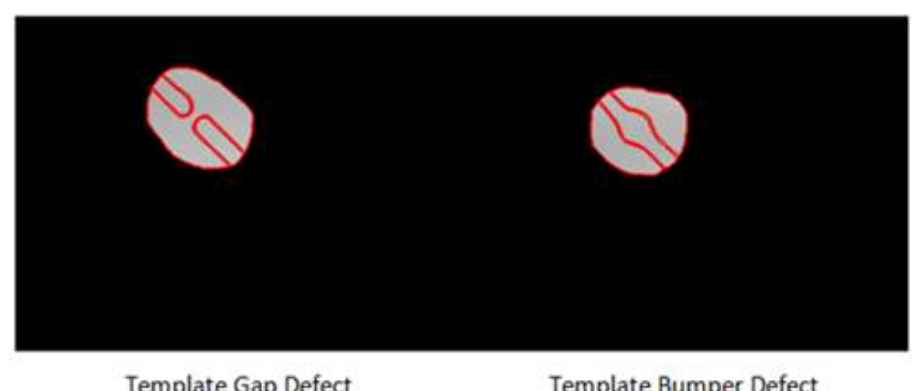

Figure 3. Template image for each defects

\section{TEMPLATE MATCHING}

Template Matching is a procedure in computerized picture handling for discovering little pieces of a picture which match a template picture. From the boundary pattern available, a direct template matching algorithm can be applied. However, such method requires huge amount of computation. In order to minimize it, the conversion of 2D image object location into single dimension must be performed. Therefore, centroid features are proposed.

\subsection{Centroid Profiles}

Centroid is the center of gravity of the object's shape. From the geometric coordinate values (x,y) of the boundary pattern, the centroid can be calculated using average values of these coordinate values. The centroid algorithm is shown in (7). The centroid for x-plane and y-plane is calculated separately and the resulting value will be center coordinate.

$$
X=\frac{x 1+x 2+\cdots+x k}{k}
$$

The centroid of a finite set of points $\mathrm{x} 1, \mathrm{x} 2, \ldots, \mathrm{xk}, \mathrm{x}$ is where $\mathrm{k}$ is the number of pixels. In template matching, model template played an important role in classifying each defect. Without proper characteristics of defects, the matching process ends with no results. Therefore, the planning of the system should integrate with both features combining in providing better recognition rate. 


\section{DISCUSSION OF RESULTS}

The points of this work are to show an adaptable visual framework for shape based matching. Test results are utilized to check the proposed methodology. In this test, three imperfections models and their relating tests are utilized to look at this methodology. Table 1 demonstrates the comparing results as indicated by 10 tried pictures utilized. Each tried picture has its own deformity to be perceived by this framework. Every one of the information results are recorded into Table 1.

Figure 4 shown to express the step by step results in this system. Figure 4(a) to Figure 4(c) shows the result of each defect model having through the 4 steps of training phase. Each defect model has its own characteristic in classifying defects. Figure 4(d) to Figure 4(f) shows the result by matching model defects on the tested images. By using template matching, each pixel of the model defects is compared with tested images in finding the exact similarity with it. Figure 4(g) to Figure 4(i) shows the gray histogram of each model defect.

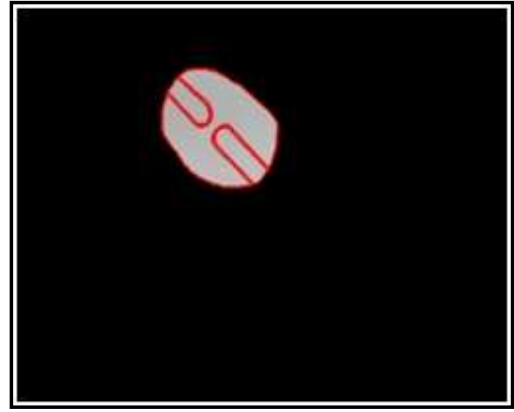

(a)

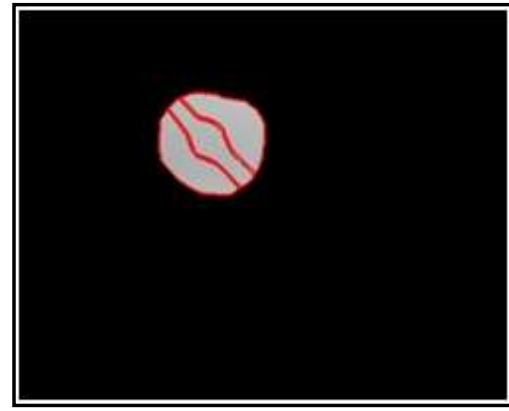

(b)

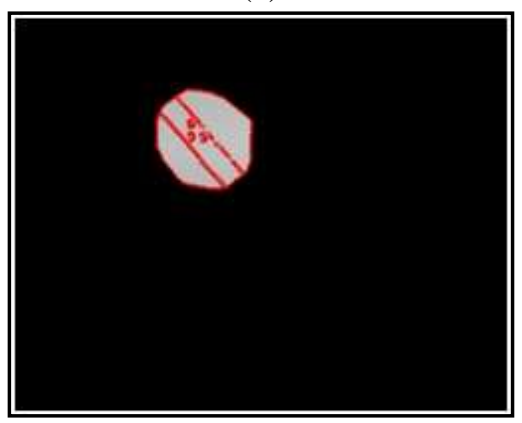

(c)

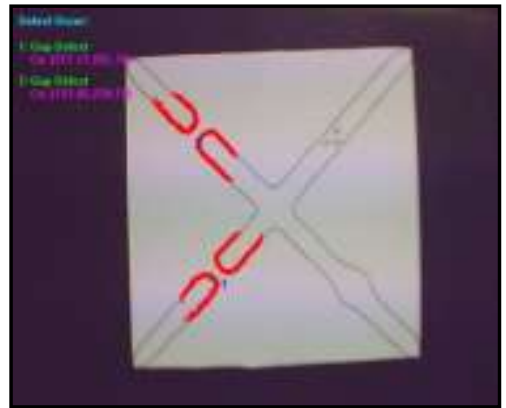

(d)

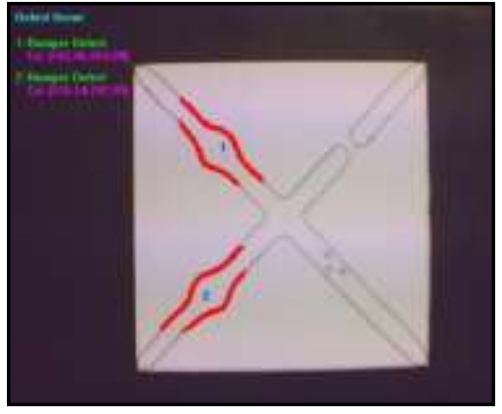

(e)

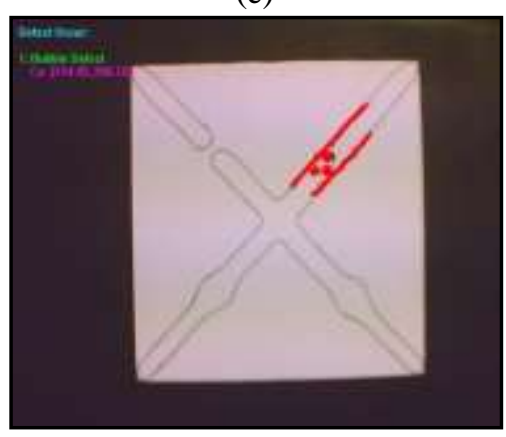

(f)

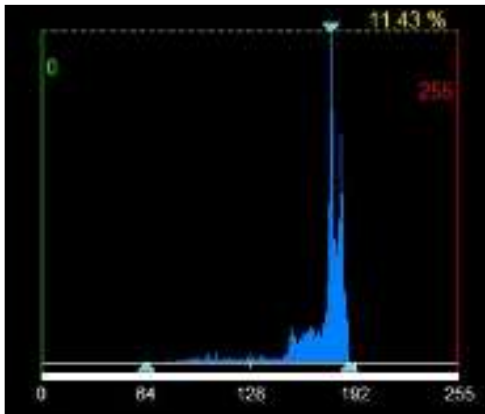

(g)

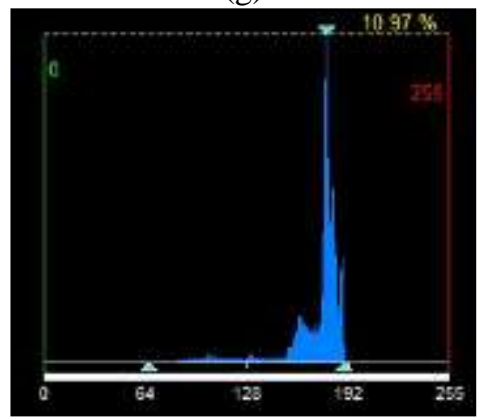

(h)

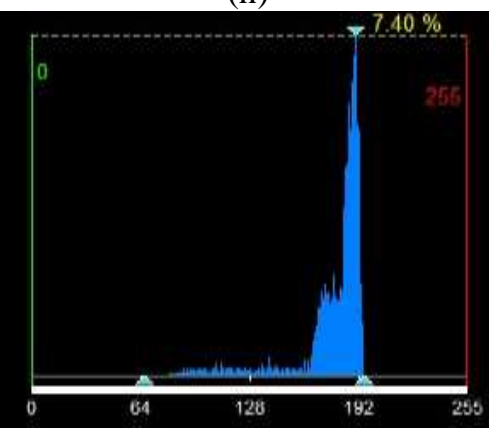

(i)

Figure 4. Result of each defect from model template, template matching and gray histogram

Table 2 shows the result of defect matching by using 10 tested images. According to the results, the recognition rate of the experiment is about $95.77 \%$ based on 3 model defects created through the system. This result is compared with [11] where it considered the problem in detecting welding defects in welding lines where the past researchers put an effort on more complex algorithm or limited in efficiency. 


\begin{tabular}{cccc}
\multicolumn{4}{c}{ Table 2. Matching Results from 10 Tested Images } \\
\hline Image & Total Defect & Defect Detected & Defect Detected $(\%)$ \\
\hline 1 & 8 & 8 & 100 \\
2 & 7 & 7 & 100 \\
3 & 8 & 7 & 87.5 \\
4 & 7 & 6 & 85.71 \\
5 & 8 & 8 & 100 \\
6 & 6 & 6 & 100 \\
7 & 7 & 7 & 100 \\
8 & 8 & 8 & 100 \\
9 & 5 & 5 & 100 \\
10 & 7 & 6 & 85.71 \\
Total & 71 & 68 & 95.77 \\
\hline
\end{tabular}

\section{CONCLUSION}

As a conclusion, auto recognition of sticking deformities is a helpful research with solid application foundation. In this paper, a technique that is utilized to concentrate highlights of imperfections and auto perceives deformities are advanced. Trial results demonstrate that the acknowledgment rate accomplished about $95.77 \%$ that greatly improved for gaining deformity discovery in sticking lines. For the most part, the technique for acknowledgment and order of imperfections in sticking lines are compelling. Furthermore, it would much be able to decrease the working exertion of person and increment the imperfection acknowledgment proficiency.

\section{ACKNOWLEDGMENTS}

We wish to express our gratitude to MOHE, Universiti Teknikal Malaysia Melaka (UTeM) especially for Centre for Robotics and Industrial Automation (CeRIA), Centre of Research and Innovation Management (CRIM) and to Faculty of Engineering Technology from UTeM to give the financial (RAGS/1/2015/TK0/FTK/03/B00114) as well as moral support for complete this project successfully.

\section{REFERENCES}

[1] L. W. Teck, et al., "Flexible approach for Region of Interest creation for shape-based matching in vision system", 2010, pp. 205-208.

[2] C. Gu, et al., "Recognition using regions," 2009

[3] D. Naso, et al., "A fuzzy-logic based optical sensor for online weld defect-detection," Industrial Informatics, IEEE Transactions on, vol. 1, pp. 259-273, 2005.

[4] A. C. Berg, et al., "Shape matching and object recognition using low distortion correspondences," 2005.

[5] X. Xie, "A review of recent advances in surface defect detection using texture analysis techniques," Electronic Letters on Computer Vision and Image Analysis, vol. 7, 2008, pp. 1-22.

[6] Sulaiman, M., M. Shah, et al. "A 3D Gluing Defect Inspection System Using Shape-Based Matching Application from Two Cameras." International Review on Computers \& Software 8(8), 2013, pp. 1997-2004.

[7] Sulaiman, M., H. N. M. Shah, et al. "Defect Inspection System For Shape-Based Matching Using Two Cameras." Journal of Theoretical \& Applied Information Technology 61(2), 2014, pp. 288-297

[8] L. Teck, et al., "Implementation of Shape-Based Matching Vision System in Flexible Manufacturing System," Journal of Engineering Science and Technology Review, vol. 3, pp. 128-135, 2010.

[9] H. M. Haniff, M. Sulaiman, et al., " Shape-based matching: Defect inspection of glue process in vision system," IEEE Symposium on Industrial Electronics and Applications (ISIEA), 2011, pp. 53-57.

[10] M. H. Harun, M. F. A. Halim, et. Al., "A 3D supervised shape-based matching approach using 2D images for defect detection," ARPN Journal of Engineering and Applied Sciences, 13 (7), 2018, pp. 2527-2534

[11] X. Xu, et al., "HALCON application for shape-based matching," 2008, pp. 2431-2434.

[12] T. Takahashi, et al., "Planning of Multiple Camera Arrangement for Object Recognition in Parametric Eigenspace, "Proceedings of the 18th International Conference on Pattern Recognition (ICPR'06), vol. 1, pp. 603-606, 2006.

[13] R. Da Silva, et al., "Radiographics pattern recognition of welding defects using linear classifiers," Insight, vol. 43, pp. 669-74, 2001.

[14] T. Liao, et al., "Detection of welding aws from radiographic images with fuzzy clustering methods," Fuzzy sets and Systems, vol. 108, p. 158, 1999.

[15] N. Nacereddine, et al., "Weld defect detection in industrial radiography based digital image processing."

[16] C. Chan and G. K. H. Pang, "Fabric defect detection by Fourier analysis," Industry Applications, IEEE Transactions on, vol. 36, pp. 1267-1276, 2000.

[17] A. Kumar and G. K. H. Pang, "Defect detection in textured materials using Gabor filters," Industry Applications, IEEE Transactions on, vol. 38, pp. 425-440, 2002. 
[18] A. Latif-Amet, et al., "An efficient method for texture defect detection: sub-band domain co-occurrence matrices," Image and Vision Computing, vol. 18, pp. 543-553, 2000.

[19] D. Mery and M. A. Berti, "Automatic detection of welding defects using texture features," Insight-Non-Destructive Testing and Condition Monitoring, vol. 45, pp. 676-681, 2003.

[20] J. Peng, "A method for recognition of defects in welding lines," in International Conference on Artificial Intelligence and Computational Intelligence, 2009, pp. 366-369.

[21] D. Naso, et al., "A fuzzy-logic based optical sensor for online weld defect-detection," Industrial Informatics, IEEE Transactions on, vol. 1, pp. 259-273, 2005.

[22] R. Meylani, et al., "Texture defect detection using the adaptive two-dimensional lattice filter," pp. 165-168 vol. 3.

[23] J. L. Sobral, "Optimised filters for texture defect detection," 2005, pp. III-565-8.

[24] W. Yigang, et al., "A Quick Algorithm to Track Welding Line Based on Computer Vision," in Second International Symposium on Computational Intelligence and Design, 2009, pp. 306-309.

[25] J. Peng, "A method for recognition of defects in welding lines," International Conference on Artificial Intelligence and Computational Intelligence, pp. 366-369, 2009.

\section{BIOGRAPHIES OF AUTHORS}

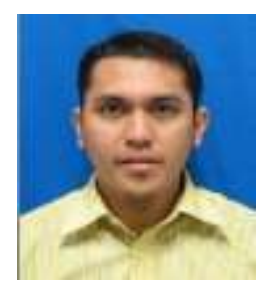

Mohamad Haniff Harun received the B. Eng (2010) and MSc (2013) in Mechatronic Engineering from Universiti Teknikal Malaysia Melaka. He is also a Senior Lecturer at Faculty of Electrical \& Electronic Engineering Technology, Universiti Teknikal Malaysia Melaka (UTeM). His primary interests related to vision system, mechatronic system, robotic application and heat transfer.

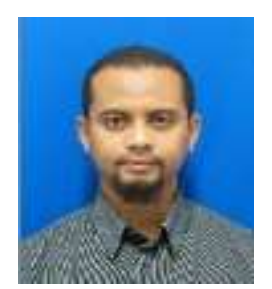

Muhamad Faizal Yaakub received his BEng in Electronics Engineering (2004) and MEng in Electrical Power Engineering (2013) form Universiti Teknologi Malaysia and Universiti Tun Hussein Onn Malaysia respectively. He is registered with Engineering Council UK as a Chartered Engineer (CEng) and with Malaysian Board of Technology as a Professional Technologist (Ts). Currently, he is a lecturer and researcher at Universiti Teknikal Malaysia Melaka. His primary interests related to power electronics, inverters and renewable energy.

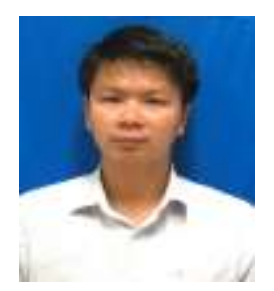

Amar Faiz Zainal Abidin received his first degree, M.Eng. (Hons) in Electrical and Electronics Engineering from University of Nottingham, Malaysia and M.Eng. from Universiti Teknologi Malaysia (UTM). He is currently attached as an academic staff with Universiti Teknikal Malaysia Melaka (UTeM). His research interests include multiobjective optimisation and natureinspired optimisation algorithms.

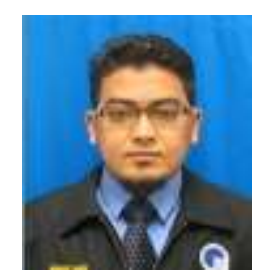

Arman Hadi Azahar received the B. Eng (2010) and MSc (2013) in Mechatronic Engineering from Universiti Teknikal Malaysia Melaka. Currently, he is a lecturer at the Universiti Teknikal Malaysia Melaka. His primary interests related to control system and mechatronic engineering.

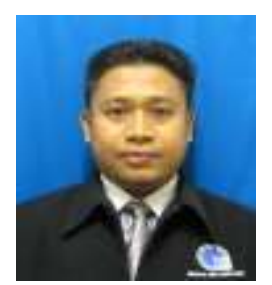

PM Dr. Mohd Shahrieel b Mohd Aras is Associate Professor at Faculty of Electrical Engineering, Universiti Teknikal Malaysia Melaka UTeM. His current research is focusing on control system design of underwater robotics and technology. His primary interests related to underwater robotics, sensory system and Artificial Intelligence techniques and system identification. 


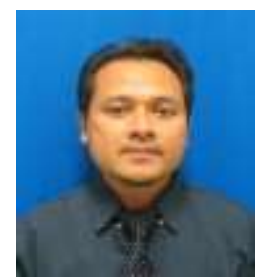

Mohd Badril Nor Shah received the M. Eng. degree in Mechatronic and Automatic Control, and the Ph.D. Degree in Electrical Engineering (Control) from Universiti Teknologi Malaysia, Johor Bahru, Malaysia, in 2011 and 2015, respectively. He also has several years of experience as an Engineer in building and facility maintenance engineering. Currently, he is a senior lecturer at Faculty of Electrical \& Electronic Engineering Technology, Universiti Teknikal Malaysia Melaka. His research interests include networked control system, real-time control system, robust control, and controller area network (CAN)

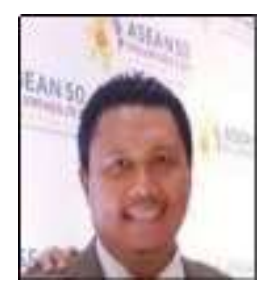

Mohd Farriz Basar is a Senior Lecturer at Faculty of Electrical \& Electronic Engineering Technology, Universiti Teknikal Malaysia Melaka (UTeM). His primary interests related to renewable energy, energy conversion and vision system. 